\title{
EFECTOS DE LA FRAGMENTACIÓN DE HÁBITATS Y PÉRDIDA DE CONECTIVIDAD ECOLÓGICA DENTRO DE LA DINÁMICA TERRITORIAL
}

\author{
Mikel GURRUTXAGA SAN VICENTE \\ Departamento de Geografía, Prehistoria y Arqueología. Universidad del País Vasco. \\ Departamento de Medio Natural y Sistemas de Información Geográfica. IKT, S.A. \\ Pedro José LOZANO VALENCIA \\ Departamento de Geografía, Prehistoria y Arqueología. Universidad del País Vasco
}

\begin{abstract}
RESUMEN: Se realiza una revisión acerca de los efectos sobre la vida silvestre que desencadenan los procesos de fragmentación derivados de las dinámicas de transformación del paisaje. Se destaca la importancia de incorporar criterios de prevención y corrección de los procesos de pérdida de conectividad del paisaje dentro de la planificación integral y sectorial de la matriz territorial en la que insertan las áreas protegidas, con objeto de optimizar la eficacia de las políticas de conservación de la naturaleza.
\end{abstract}

PALABRAS CLAVE: Fragmentación de hábitats, homogeneización del paisaje, conectividad ecológica, conservación.

ABSTRACT: A review on the effects on the wildlife that unleash the processes of fragmentation derived from the dynamics of transformation of the landscape is realized. It is outlined the importance of incorporating criteria of prevention and correction of the processes of loss of landscape connectivity inside the integral and sectorial planning of the territorial matrix in which they insert the protected areas, in order to optimize the efficiency of the conservation policies.

KEY WORDS: Habitat fragmentation, landscape homogenization, ecological connectivity, conservation..

$\boldsymbol{R} \boldsymbol{E} \boldsymbol{S U} \boldsymbol{M} \boldsymbol{E}:$ Cet article révise les effets que déchaînent les processus de fragmentation, dérivés des dynamiques de transformation du paysage, sur la vie sauvage. On signale l'importance d'intégrer des critères de prévention et correction des processus de perte de connectivité du paysage dans la planification intégrale et sectorielle de la matrice territoriale dans où s'insèrent les zones protégées, afin d'optimiser l'efficacité des politiques de conservation de la nature.

MOTS-CLÉS: Fragmentation d'habitats, homogénéisation du paysage, connectivité écologique, conservation.

RESUMO: No artigo atual uma revisão é feita sobre os efeitos na vida selvagem que provocam os processos da fragmentação derivados dos dinâmicos da transformação da paisagem. A importância está fora dos critérios incorporando da prevenção e da correção dos processos da perda do conectividade da paisagem dentro do planeamento integral e sectorial da matriz territorial em que introduzem as áreas protegidas, com objeto para optimize a eficácia das políticas do conservação da natureza.

PALAVRAS-CHAVES: Fragmentação de habitat, homegeneização da paisagem, conectividade ecológica, conservação. 


\section{INTRODUCCIÓN}

Las principales tendencias que se han producido en la dinámica de los paisajes europeos durante las últimas décadas son la reducción y fragmentación de hábitats naturales y la homogeneización de los paisajes agrícolas (JONGMAN, 2002). Ambas tendencias, estrechamente relacionadas entre sí, se han producido por profundos cambios en los usos del suelo y en las prácticas de aprovechamiento de los agrosistemas (DÍAZ PINEDA, 2001). Así, presentan una vital importancia las actividades antrópicas, manifestadas en los usos del suelo del territorio y en las prácticas de manejo de los ecosistemas, como agentes moldeadores de los paisajes humanizados, en general, y de los procesos de fragmentación, en particular.

La fragmentación se manifiesta, no sólo como proceso, sino como patrón dentro del espacio geográfico. Es por esto que la disposición espacial de los fragmentos de hábitat en el territorio influye sobre la movilidad de las especies silvestres entre los mismos y, por ende, sobre la dinámica de poblaciones. Esto enlaza con el objeto de estudio de una disciplina de síntesis, como es la ecología del paisaje. Ésta, que se basa fundamentalmente en la puesta en común de la visión horizontal de la geografía y la vertical de la ecología, integra el estudio de las relaciones entre las variaciones de los patrones espaciales del paisaje con sus agentes causales y sus consecuencias sobre los procesos ecológicos.

De esta manera, se da una saludable encrucijada de puntos de vista entre diferentes ciencias naturales (biología, ecología) y ciencias sociales (historia, geografía) en el estudio de la gestión de los ecosistemas y en el análisis de la evolución de la estructura de los paisajes, entendiendo éstos como el resultado de la interacción de las sociedades humanas con el medio en el que habitan (BUREL Y BAUDRY, 2002). Así, y dado que las condiciones de partida y las dinámicas del pasado son determinantes en la comprensión del estado y funcionamiento de los ecosistemas naturales, así como en el estudio de su evolución futura, disciplinas como la prehistoria y la arqueología (con sus aplicaciones en la biogeografía histórica), por un lado, y la etnografía, la antropología, la geografía humana y la agronomía, por otro, se encargan, respectivamente, del estudio de la formación de los paisajes y de las técnicas aplicadas por las sociedades para transformarlos y moldearlos.

Dentro de las consecuencias sobre los procesos ecológicos derivadas de variaciones de los patrones espaciales del paisaje, se encuentran las que sobre la vida silvestre se producen por efecto de procesos de fragmentación de hábitats. Éstos, en términos funcionales se traducen en pérdidas de conectividad ecológica, entendida como la capacidad con la que cuenta el paisaje para permitir los desplazamientos de determinadas especies o poblaciones entre las teselas con 
recursos (TAYLOR ET AL., 1993). En este sentido, la conectividad ecológica se hace garante del mantenimiento de una serie de procesos vitales entre los que destacan los desplazamientos de los organismos para alimentarse, refugiarse, reproducirse o dispersarse. Sin ellos, la pervivencia de la mayor parte de los organismos silvestres se ve seriamente comprometida.

En la medida en que los procesos de reducción y fragmentación de hábitats son apuntados por la comunidad científica como una de las principales causas, si no la principal, de la actual crisis de biodiversidad (PRIMACK, 2002; FAHRIG, 2003; CROOKS Y SANJAYAN, 2006), se buscan y plantean soluciones aplicadas para hacer frente a esta problemática, de forma que la conservación y, en su caso, restauración de la conectividad del paisaje conforma una de las principales líneas de investigación y planificación en la actualidad.

En este contexto, el desarrollo de redes de conservación funcionales -también denominadas redes ecológicas (MUGICA ET AL., 2002)-, planteadas bajo la premisa de conservar la conectividad ecológica territorial mediante el establecimiento de corredores ecológicos entre áreas protegidas, es aún incipiente y en los últimos años ha atravesado una etapa fundamentalmente teórica (DE LUCIO ET AL., 2003)

Derivada de esta necesidad, que se debe traducir en la generación de un marco normativo específico y unas figuras de planificación claras y coordinadas a diferentes niveles con objeto de preservar la conectividad del paisaje, se requiere una primera revisión que analice cuáles son los diferentes efectos que los procesos de fragmentación presentan sobre las comunidades y poblaciones silvestres.

\section{EFECTOS DE LA FRAGMENTACIÓN SOBRE LA VIDA SILVESTRE}

\section{II.1. Efectos sobre la composición y riqueza de las comunidades biológi- cas}

En general, se derivan ciertas tendencias en cuanto a la composición de las comunidades biológicas en paisajes fragmentados. Por un lado, la reducción de los hábitats naturales o seminaturales, favorece el incremento demográfico de especies generalistas y el descenso de las especialistas (GASCON ET AL., 1999). Además, diversos autores han constatado que la homogeneización y fragmentación del paisaje provoca un mayor incremento de las especies invasoras (RIVARD ET AL., 2000, WITH ET AL., 2002; BAKKER Y WILSON, 2004). En el extremo opuesto, en las grandes reservas naturales, cada vez más escasas a nivel planetario, las especies asociadas a los hábitats de interior bien conservados representan la mayor parte de los organismos de la comunidad biológica. 
La Teoría de la Biogeografía de Islas (MACARTHUR Y WILSON, 1967) supuso una primera aproximación a la dinámica de la composición de las comunidades en fragmentos. La evidencia de que, para un hábitat homogéneo, contienen menos especies las islas alejadas del continente y las ínsulas menores respecto a las de mayor superficie, constituyó el punto de partida de esta teoría. Concretamente, propone que el número de especies que forman la comunidad biológica de una isla tiende a un equilibrio dinámico determinado por un balance entre la tasa de colonización de nuevas especies y la tasa de extinción de taxones residentes en la misma. La tasa de colonización depende fundamentalmente del distanciamiento de la isla respecto al continente, mientras la tasa de extinción de especies es mayor cuanto menor es el área de la ínsula. Su modelo se extrapoló al caso de los hábitats naturales del continente en paisajes fragmentados, por tratarse de manchas rodeadas de hábitats de carácter antrópico, funcionando como "islas" naturales en un "mar" de espacios transformados. No obstante, es preciso señalar que actualmente la teoría insular se considera desfasada, especialmente en el plano de la gestión ambiental, dado que parte de un modelo espacial simplificado que no considera la heterogeneidad del paisaje y otras variables como la calidad del hábitat.

Con base en la teoría insular, DIAMOND (1975) propuso una serie de reglas para el diseño de redes de reservas, fundamentadas en que una reserva grande preserva más especies que varias menores, y en que las reservas deben encontrarse lo más cerca posible unas de otras. Esto abrió un amplio debate durante los años 1970 y 1980 que se dio en denominar SLOSS (Single Large or Several Small). Los opositores argumentaban que es posible encontrar un mayor número de especies en varias reservas menores separadas geográficamente, dada la mayor diversidad de hábitats (SIMBERLOFF Y ABELE, 1976; SiMBERLOFF, 1982). No se consiguió alcanzar un consenso, debido a que no existe un patrón universal por el que se pueda validar una determinada regla al respecto. El debate SLOSS constituyó el caldo de cultivo para que en 1979 se diera inicio al mayor proyecto de investigación sobre los efectos de la fragmentación forestal que se realiza en el mundo. Se trata del proyecto Dinámicas biológicas en fragmentos forestales (Biological Dynamics of Forest Fragments Project, BDFFP) que, sustentado por el Instituto Smithsoniano de Investigación Tropical de Panamá y el Instituto Nacional para la Investigación Amazónica de Brasil, desarrolló el biólogo Lovejoy planteando el seguimiento de las dinámicas en fragmentos boscosos de diferentes tamaños. En un principio el proyecto se denominó "Mínimo tamaño crítico de ecosistemas". Después de 26 años de investigación, el macroproyecto ha dado lugar a notables y numerosas aportaciones (véase para revisiones LAURANCE Y BIERREGAARD, 1997; BIERREGAARD ET AL., 2001). 
Volviendo a las bases de la teoría insular, es preciso destacar que la relación especies-área, que responde a una mayor riqueza a medida que aumenta el tamaño de una mancha de hábitat (PRESTON, 1962), constituye una de las reglas ecológicas más estudiadas y consensuadas (ROSENZWEIG, 1995). No obstante, se ha apuntado que esta relación especies-área podría cumplirse de forma genérica a partir de una superficie mínima dada, teniendo en cuenta que en los parches más pequeños la riqueza variaría independientemente de la superficie, fenómeno que se ha denominado efecto de las islas pequeñas (LOMOLINO, 2000). Según LOMOLINO Y WEISER (2001) el tamaño crítico de las manchas a partir del cual la riqueza varía con la superficie es genéricamente mayor en mamíferos no voladores, intermedio en aves, reptiles y coleópteros, y menor en plantas.

En general, los animales de mayor tamaño requieren de dominios vitales más extensos que los de menor, los carnívoros más amplios que los herbívoros, los endotermos mayores que los ectotermos y los gregarios superiores a los solitarios. De esta manera, la reducción de los bosques provoca procesos de extinción local en cadena, comenzando por las especies forestales que presentan menor densidad poblacional por unidad de supercie (TELLERÍA Y SANTOS, 1999; TELLERÍA, 2001). Por ejemplo, DÍAZ ET AL. (2000) comprobaron en dos sectores de la meseta septentrional ibérica que la lagartija colilarga (Psammodromus algirus) se encuentra en fragmentos boscosos de un tamaño mínimo de 90 ha. Sugieren que la mortalidad supera a la natalidad en los fragmentos más pequeños, debido fundamentalmente a la mayor tasa de predación sobre la especie debido al efecto borde, el cual se explica posteriormente.

Todo ello enlaza, además de con la noción de área mínima viable de las especies (SHAFFER, 1987), con el concepto de los umbrales ecológicos (ecological thresholds) (HUGGETT, 2005). En el campo de estudio de los procesos de fragmentación y pérdida de conectividad poblacional, un umbral sucede cuando la respuesta de una especie o un grupo de especies no es lineal a la pérdida del hábitat, sino que cambia abruptamente a cierto nivel de pérdida (TURNER Y GARDNER, 1991; WITH Y CRIST, 1995; WITH Y KING, 1999; TOMS Y LESPERANCE, 2003; MANDUJANO Y ESTRADA, 2005; LUCK, 2005; RADFORD ET AL., 2005; SCHULTZ Y CRONE, 2005). De esta forma, la identificación de umbrales críticos de fragmentación y distancia de aislamiento para las especies ha de ser una de las prioridades de investigación en territorios humanizados (DE LUCIO ET AL., 2003), en la medida en que en base a ellos se pueden establecer prioridades para desarrollar acciones de conservación de especies o grupos de especies, así como optimizar los recursos necesarios para su ejecución (RADFORD ET AL., 2005). 
Se ha detectado además un patrón general en base al cual la densidad de individuos de las especies asociadas a un hábitat disminuye a medida en que el tamaño de fragmento de éste se reduce (CONNOR ET AL., 2000; BOWMAN ET AL., 2002; TISCHENDORF ET AL., 2005), superando así los modelos simplificados en los que la densidad de una población se mantiene constante, al crecer el número de individuos linealmente con el área disponible. En general la relación densidad-área estaría condicionada fundamentalmente por el balance emigracióninmigración en los parches a medida que varía el tamaño de éstos, de forma que en función de la sensibilidad de la especie a los bordes (TISCHENDORF ET AL., 2005), de su capacidad dispersiva y comportamiento inmigratorio (BOWMAN ET AL., 2002) dicha relación puede ser positiva o negativa (DEBINSKI Y HOLT, 2000; BENDER ET AL., 1998).

Sin perjuicio de lo anterior, de cara a la comprensión global de la composición de las comunidades biológicas en el paisaje, ha de considerarse el papel de la heterogeneidad del mosaico paisajístico. Dentro de éste, numerosas especies hacen uso de diversos tipos de parches para completar sus ciclos vitales. Esto enlaza con el concepto de unidad funcional, definido por MERRIAM (1984) como el conjunto de elementos paisajísticos utilizados por una especie en su ciclo de vida. Así, las especies multihábitat, que son aquellas que requieren de diferentes elementos paisajísticos para realizar sus funciones y completar su ciclo vital, se asocian necesariamente a paisajes heterogéneos (LAW Y DICKMAN, 1998). Además, la coexistencia de distintos grupos de especies, asociados a diferentes biotopos, es una circunstancia que incrementa la biodiversidad global y que se produce en paisajes estructuralmente heterogéneos (PINO ET AL., 2000; ATAURI Y DE LUCIO, 2001). De hecho, la teoría de la heterogeneidad del hábitat predice que una mayor heterogeneidad del paisaje se asocia con una mayor biodiversidad, al existir una mayor diversidad de tipos de hábitats (WILLIAMS, 1964), de forma que en general las áreas mayores, al contener un rango más amplio de diferentes recursos, presentan mayor riqueza de especies que las menores. No obstante, esto no siempre es cierto ya que la disposición y las características de los elementos del mosaico paisajístico, además de factores históricos y biogeográficos, tienen un papel decisivo en la configuración de las comunidades biológicas.

En el caso de paisajes heterogéneos en los que se asientan diferentes usos extensivos y áreas de vegetación climácica, la biodiversidad asociada suele ser muy elevada, incluso mayor que la de extensos espacios inalterados. Por ejemplo, los paisajes mediterráneos albergan una gran diversidad de especies debido a la diversidad fisiográfica y climática, a las dinámicas históricas y a la coexistencia de espacios seminaturales creados y mantenidos por usos agroganaderos 
tradicionales con áreas y retazos de vegetación natural (GONZÁLEZ BERNÁLDEZ, 1981; FARINA, 1998; BLONDEL y AROSON, 1999).

Así, la homogeneización de los paisajes agrarios, como consecuencia de la intensificación de los aprovechamientos, ha provocado la pérdida de numerosas especies asociadas a los agrosistemas tradicionales (TUCKER, 1997; DONALD, 2004). También el abandono de la actividad agrícola en áreas menos productivas causa, en numerosos casos, la disminución de la diversidad biológica asociada al territorio (GONZÁLEZ BERNÁLDEZ, 1991; NAVEH, 1994; DÍAZ ET AL., 1998; SÚAREZ-SEOANE ET AL., 2002), al existir una numerosa comunidad de organismos adaptados a los agrosistemas estructuralmente heterogéneos.

No obstante, niveles altos de heterogeneidad pueden estar asociados a un elevado grado de fragmentación de los hábitats y a la consiguiente presencia de manchas muy pequeñas incapaces de albergar poblaciones viables de ciertos organismos. Así, el incremento de la diversidad asociado a la heterogeneidad puede presentar un valor máximo por encima del cual empiece a disminuir (SANTOS Y TELLERÍA, 1998).

\section{II.2. Efecto borde sobre los fragmentos de hábitat}

Fruto de los procesos de fragmentación, el aumento de la relación perímetro/superficie de los fragmentos de hábitat en el paisaje provoca la mayor permeabilidad de éstos ante la influencia de los ambientes periféricos. Los diversos procesos asociados que inciden sobre las dinámicas y condiciones que tienen lugar en los fragmentos son conocidos como "efecto borde" (MURCIA, 1995; LAURANCE, 1997; WOLF Y BATZLI, 2002; DESROCHERS ET AL., 2003; SCHTICKZELLE Y BAGUETTE, 2003).

El efecto borde decrece conforme aumenta la distancia hacia el interior del fragmento, aunque de manera diferente en cada caso. La importancia de las alteraciones ecológicas causadas por el efecto borde sobre los fragmentos es modulada por el carácter más o menos abrupto del ecotono, lo cual depende en buena medida del grado de contraste entre el hábitat fragmentado y los medios que le rodean. Así, los efectos en fragmentos embebidos dentro de una matriz estructuralmente similar al hábitat original serían menores a los inmersos en un entorno hostil para la biota asociada al hábitat del fragmento (LINDENMAYER Y FRANKLIN, 2002). En función del grado de contraste estructural y condiciones de naturalidad, se diferencian las transiciones entre hábitats de "borde duro" y "borde blando" (WIENS ET AL., 1985). Las primeras poseen un elevado contraste y son más frecuentes en paisajes transformados, mientras las segundas presentan cambios graduales y una mayor transitabilidad por especies de fauna silvestre. 
Así, a pesar del aumento de la heterogeneidad ambiental que introducen en el paisaje los ecotonos o zonas de transición entre ecosistemas, que poseen flora y fauna características y pueden actuar como una comunidad funcional distinta de las otras dos que contactan (ODUM, 1958), cuando presentan una elevada proporción en superficie respecto al hábitat de interior, éste sufre procesos de degradación progresiva por efectos de borde a diferentes niveles. En pequeños fragmentos el efecto borde llega a afectar a la totalidad de la superficie de los mismos. Por todo ello, la proporción de superficie de borde en relación al área de los fragmentos tiene gran importancia en el efecto global que la fragmentación provoque sobre el hábitat.

En primer lugar, cabe destacar que la fragmentación de los bosques provoca modificaciones en el microclima interno (DIDHAM Y LAWTON, 1999), por incremento de la insolación y de la exposición al viento, a las lluvias y a las heladas en los fragmentos. Como consecuencia, los regímenes de humedad y temperatura se ven alterados en los fragmentos boscosos (LOVEJOY ET AL., 1986). Estos cambios tienen efectos sobre la estructura y composición de la vegetación, con las consiguientes repercusiones sobre la comunidad faunística asociada (PALIK Y MURPHY, 1990; BALDE, 1999). Ésta se ve afectada tanto por la variación de los recursos vegetales disponibles como por el aumento de la vulnerabilidad ante los cambios climáticos.

Asimismo los bordes pueden afectar a las interacciones bióticas. El predominio de las áreas de borde incide sobre las interacciones entre las especies de la comunidad biológica (FAGAN ET AL., 1999; MATLACK Y LITVAITIS, 1999). A través de los bordes los fragmentos pueden recibir la penetración de organismos generalistas y la invasión de especies exóticas, así como sustentar una mayor presencia de especies asociadas al propio ecotono o a la matriz. Así, fruto del efecto borde, plantas invasoras pueden llegar a desplazar a especies nativas que proveen alimento a la fauna forestal. Además, derivada de la presencia de especies invasoras animales, la mayor competencia por los recursos alimenticios, como frutos y semillas, puede dañar la capacidad de regeneración de la vegetación del fragmento así como comprometer la supervivencia de especies especialistas del hábitat por exclusión competitiva (BARRETT ET AL., 1994). A su vez, al aumentar la proporción de bordes en los fragmentos de hábitat, aumenta la accesibilidad de especies depredadoras que habitan en la periferia sobre aquellos taxones faunísticos de interior que constituyen presas para aquellas. Estas últimas son algunas de las razones por las que determinados autores también han puesto en cuestión el diseño de corredores más o menos lineales o estrechos entre manchas de hábitat (DEL BARRIO, 2000; DE LUCIO, 2003).

Por otro lado, en algunos estudios se ha comprobado un descenso en el éxito reproductor en los bordes de fragmentos forestales, al ser mayor la depredación 
de huevos y el parasitismo de nidos (WILCOVE, 1985; ANDRÉN Y ANGELSTAM, 1988; WILLSON ET AL., 2001), si bien en otros estudios no se han detectado diferencias entre bordes e interior (véanse ANDRÉN, 1995; CHALFOUN ET AL.; 2002 Y STEPHENS ET AL., 2003 para revisiones). Las características estructurales del borde afectan en gran medida a la tasa de predación de nidos, de forma que los de vegetación más densa presentan mejores tasas de éxito reproductor (HARTLEY Y HUNTER, 1998).

Como consecuencia de estas interacciones biológicas pueden producirse diversos efectos secundarios en cadena. Por ejemplo, la reducción poblacional de organismos polinizadores y dispersadores de semillas puede afectar a la subsistencia de los fragmentos a largo plazo. Asimismo, la menor abundancia de aves insectívoras puede conllevar un aumento de insectos fitófagos y el incremento de los índices de defoliación de las plantas.

Entre las actividades antrópicas que se desarrollan en la periferia de fragmentos de hábitat que pueden producir efecto borde sobre éstos, se encuentran aprovechamientos agrícolas intensivos, en la medida que provocan la contaminación del suelo mediante el uso los fertilizantes y fitosanitarios. Además, la ocurrencia de plagas en los fragmentos puede aumentar por su proximidad a las zonas agrícolas. El ganado, por su parte, puede afectar a la regeneración de las plantas situadas en el borde del fragmento por la fuerte presión que ejerce sobre yemas, frutos y semillas. Tanto es así, que en fragmentos residuales de escasa superficie el conjunto de herbívoros silvestres y domésticos puede consumir la totalidad de la producción de frutos y semillas. En cuanto a la frecuentación de personas que acceden a la periferia o interior de los fragmentos, actividades como la tala de ramas y troncos, las molestias a la fauna, el furtivismo y el abandono de basuras contribuyen a la degradación del hábitat. El impacto de estas actividades fluctúa dependiendo de la época en que se lleven a cabo. Así, sería máximo en época de celo y cría y mínimo, en periodo de hibernada o reposo.

\section{II.3. Efectos sobre la dinámica de poblaciones}

La pérdida de efectivos en las poblaciones y el paulatino aislamiento de éstas respecto a otros grupos de individuos son los principales factores estrechamente relacionados con los procesos de reducción y fragmentación de hábitats que causan problemas de inviabilidad a numerosas especies silvestres en paisajes humanizados.

En primer lugar, los procesos de reducción y fragmentación del hábitat provocan localmente una paulatina disminución del número de individuos en las poblaciones de organismos silvestres que dependen de él. En este sentido, los 
cambios en los patrones de abundancia producto de la fragmentación del hábitat pueden responder a fenómenos demográficos que modifican el crecimiento de las poblaciones que habitan los fragmentos (DOOLEY Y BOWERS, 1998; JULES, 1998; BRUNA Y OLI, 2005).

En este contexto, el concepto de población mínima viable (MVP, minimum viable population) (SHAFFER, 1981; GILPIN Y SOULÉ, 1986), es un estimador del número mínimo de individuos de una población aislada que tiene máximas probabilidades de sobrevivir a largo plazo, dada su capacidad de evitar o superar posibles factores negativos que pueden conducir a la especie a la extinción (estocasticidad demográfica, ambiental y genética, y catástrofes naturales). Complementariamente, el concepto de área mínima viable (MVA, minimum viable area) (SHAFFER, 1987; WHITTAKER, 1998; BROOK, 2003) se refiere a la superficie mínima de hábitat aislado que necesita la población para sobrevivir, es decir, el área que requiere la población mínima viable para mantenerse.

Más allá de la reducción del tamaño de una mancha de hábitat preexistente, ésta puede quedar dividida en varios fragmentos, de forma que a la reducción de superficie total de hábitat se le suma la discontinuidad del mismo en el espacio. Las poblaciones afectadas no sólo dispondrán de menor superficie de hábitat, sino que además tendrán que atravesar espacios transformados de la matriz para moverse entre las teselas con recursos. Así, dentro del dominio vital de la especie, ésta debe ser capaz de acceder a sus lugares de alimentación, refugio o cría y, en su caso, de realizar los desplazamientos estacionales que requiera. Asimismo la especie ha de ser capaz de realizar desplazamientos dispersivos con los que mantener ciertos niveles de intercambio genético entre poblaciones y con los que eventualmente ocupar territorios adecuados en los que asentarse. Por tanto, la conectividad ecológica, entendida como la capacidad del territorio para permitir el desplazamiento de las especies entre las teselas con recursos, se configura como un aspecto vital en la dinámica de las poblaciones fragmentadas.

Además, es preciso tener en cuenta que puede haber sectores de hábitat óptimos donde una especie prospere y sectores subóptimos donde ésta se mantenga gracias al aporte de individuos procedentes de hábitats mejores. Esta forma de interacción poblacional con el medio responde al Modelo Fuente-Sumidero propuesto por PULLIAM (1988), en base al cual la presencia de una especie en los hábitats sumidero está supeditada a que exista una inmigración continua de individuos desde los hábitats fuente.

El grado en que los procesos de fragmentación y homogeneización del paisaje afectan a la conectividad de las poblaciones determina en gran medida la viabilidad de las especies en el paisaje. Si los efectivos de una especie que habitan el 
conjunto de parches separados presentan altas tasas de desplazamiento entre los mismos, siguen formando una población. En cambio, si la permeabilidad de los espacios que rodean los fragmentos al flujo de los individuos disminuye de tal manera que éstos presentan tasas medias o bajas de dispersión, puede decirse que la población tiende a dividirse en varias subpoblaciones. En esta línea, los organismos pueden distribuirse en un conjunto de poblaciones locales que habitan en los fragmentos y que mantienen un cierto intercambio de individuos entre sí, de tal forma que muestran una distribución groseramente continua. Este modelo de distribución y de relaciones dio lugar al concepto de metapoblaciones, introducido por LEVINS (1970). Por último, si el flujo de organismos entre los fragmentos es muy bajo o nulo, cada parche contiene una población distinta.

La dinámica de metapoblaciones es el enfoque más utilizado en el estudio de las poblaciones fragmentadas, ya que la presencia de poblaciones locales distribuidas en diferentes parches conectadas por migración limitada constituye, además de un patrón común en especies asociadas a hábitats fluctuantes o con perturbaciones frecuentes, el estadío más habitual en el que se encuentran las especies afectadas por procesos de fragmentación de hábitats. Así, numerosas especies se distribuyen en grupos de poblaciones locales relativamente pequeñas que habitan manchas de hábitat separadas e interactúan a través de individuos que se mueven entre ellas. Estas poblaciones locales interactuantes forman en su conjunto una metapoblación. Si bien las poblaciones locales son por su pequeño tamaño propensas a la extinción, la metapoblación persiste mientras se mantenga un equilibrio entre extinciones locales y recolonizaciones de parches (OPDAM, 1991; HANSKI Y GILPIN, 1997; HANSKI, 1999). De esta forma, la dinámica de la metapoblación determina la persistencia regional de una especie.

Sin embargo, si se produce un descenso en los niveles de conectividad ecológica en el paisaje, el intercambio de individuos entre parches disminuye, al igual que las tasas de colonización, de forma que las poblaciones locales van quedando aisladas. La disminución de la conectividad entre las poblaciones puede deberse al aumento de la distancia entre fragmentos, a cambios en las características de la matriz, que la hacen menos permeable, o a una combinación de ambos factores. De esta forma, las poblaciones locales, que por su pequeño tamaño eran de por sí tendentes a la extinción, ven aumentada esta tendencia debido a su aislamiento. El escaso o nulo intercambio genético con otras poblaciones locales, la gran dificultad para ser suplementadas por individuos de otra población o la práctica imposibilidad para colonizar parches vacíos hacen que la metapoblación deje de funcionar como tal. Las bajas tasas de inmigración en los fragmentos impiden que se produzca el denominado efecto rescate (BROWN Y KOBRIC-BROWN, 1977), que favorece la viabilidad de las poblaciones mediante la suplantación de poblaciones reducidas y de incluso parches vacíos mediante 
individuos inmigrantes. Se llega así al estadío en que se forman poblaciones distintas, sin intercambio de individuos, lo cual las hace muy vulnerables ante factores determinísticos y estocásticos de extinción.

Por todo, el aumento de la vulnerabilidad de las poblaciones fragmentadas, afectadas por la reducción demográfica y el aislamiento genético, tiene como consecuencia última que se desencadenen procesos de extinción de especies, ya sea a escala local, regional o global. La incidencia de todo tipo de perturbaciones sobre estas poblaciones pequeñas y genéticamente mal dotadas, acelera los procesos de extinción.

Así, cuanto más pequeña (menor número de efectivos) es una población aislada, más vulnerable es. Esto se debe a que una población numerosa tiene mayor capacidad para responder a los procesos determinísticos y menor sensibilidad a los procesos estocásticos que pueden llevarla a la extinción. Los procesos determinísticos de extinción son aquellos que tienen una relación causa-efecto sobre las poblaciones y generalmente son generados por las actividades antrópicas (como la degradación del hábitat, la introducción de especies depredadoras, la caza, etc.). Los factores estocásticos de extinción, en cambio, son aleatorios e impredecibles, pudiendo dividirse en los siguientes:

- estocasticidad demográfica, que se refiere a variaciones aleatorias en parámetros poblacionales como la tasa de natalidad, la tasa de mortalidad o la sex ratio.

- estocasticidad genética, derivada de procesos genéticos aleatorios que pueden cambiar las características genéticas de la población (pérdida de variabilidad genética, incremento de las mutaciones, etc.)

- estocasticidad o incertidumbre ambiental, causada por enfermedades, por variaciones impredecibles en el clima, en los recursos alimenticios, en las poblaciones de competidores, depredadores y parásitos, etc. También dentro de este grupo aparecerían las catástrofes naturales, como incendios, inundaciones o terremotos.

Como factor de extinción notablemente fomentado por la fragmentación del hábitat cabe destacar la estocasticidad genética. Fruto de la consanguinidad y de la deriva genética en poblaciones pequeñas y aisladas, la pérdida de variabilidad genética de la población puede impedir a ésta adaptarse y responder a cambios del medio o superar problemas con los que ya se había enfrentado eficazmente en el pasado. Así, como consecuencia del alto grado de apareamientos entre individuos emparentados (cruzamientos consanguíneos) en las poblaciones pequeñas y aisladas, se modifican las frecuencias génica, genotípica y fenotípica. El problema que plantea la endogamia o consanguinidad en la población es que 
alelos recesivos deletéreos pueden alcanzar altas concentraciones y manifestarse en muchos de los individuos de la misma, produciendo una disminución de la adaptación y del rendimiento biológico. Además de la consanguinidad o endogamia, la deriva genética es otro problema genético que afecta a poblaciones pequeñas y consiste en la pérdida aleatoria de variabilidad genética. Cuando el número de efectivos es reducido, crece la probabilidad de que algunos alelos se pierdan porque ninguno de los individuos que lo poseen lo transmite a la descendencia.

Además de los problemas de viabilidad genética, en las etapas finales de los procesos de fragmentación, tal y como advierten SANTOS Y TELLERÍA (2006), se pueden producir efectos negativos sobre la condición corporal y la estabilidad durante el desarrollo de los individuos (LENS ET AL., 1999; DÍAZ ET AL., 2005). También se ocasionan descensos en la fecundidad, debido a una baja densidad poblacional y a la consiguiente dificultad o retraso de los emparejamientos, fenómeno denominado efecto Allee (ALLEE ET AL., 1949).

\section{SENSIBILIDAD A LA FRAGMENTACIÓN}

Por último, es preciso destacar que el grado de incidencia de los procesos de fragmentación del paisaje sobre la dinámica de poblaciones varía en función de la sensibilidad que presenten las especies hacia los mismos. Esta sensibilidad depende de múltiples factores relacionados con los requerimientos de hábitat y la biología de las especies. Según los estudios realizados al respecto, las especies más sensibles a la reducción y fragmentación de su hábitat y por tanto las más propensas a sufrir extinciones a diferentes escalas espaciales y temporales por este motivo poseen alguna o algunas de las siguientes características (LAURANCE, 1990; NOSS Y CUTSI, 1994; HENLE ET AL., 2004):

- Ocupan posiciones elevadas en la cadena trófica, se distribuyen en bajas densidades de individuos y requieren extensas áreas de campeo. Por ejemplo grandes mamíferos carnívoros.

- Requieren hábitats específicos, de forma que tendrán dificultades para atravesar la matriz. Por ejemplo especies estrictamente forestales no voladoras, como la marta Martes martes.

- Dependen de fuentes de alimento específico, de forma que no podrán optar por otras alternativas. Por ejemplo los insectos saproxílicos, tal y como advierte SCHIEGG (2000).

- Requieren de recursos estacionales, de forma que utilizan determinadas combinaciones de hábitats y necesitan desplazarse entre ellos. Por ejemplo ciertas aves esteparias, como la avutarda (Otis tarda). 
- Dependen de recursos fluctuantes, cuya disponibilidad puede variar en el espacio y en el tiempo. Por ejemplo los anfibios.

- Dependen de hábitats particularmente vulnerables al cambio climático. Por ejemplo el águila imperial ibérica (Aquila adalberti).

- Su distribución se limita a zonas elevadas en altitud o en latitud, de forma que dicha distribución puede tender al acantonamiento como consecuencia del cambio climático. Por ejemplo las especies nivales.

- Poseen baja capacidad de dispersión en relación a las distancias entre los fragmentos. Por ejemplo ciertos invertebrados que se desplazan por tierra o con escasa capacidad de vuelo.

- Presentan una baja fecundidad, de forma que tienen dificultades para la recuperación poblacional tras periodos regresivos y tienden a sufrir tempranamente problemas de diversidad genética. Por ejemplo el oso pardo Ursus arctos.

- Presentan un comportamiento marcadamente social, de forma que no prosperan en bajas densidades. Por ejemplo diversas especies de hormigas forestales.

- Ocupan solamente el hábitat de interior de ciertos tipos de parches. Son por tanto sensibles a los bordes y no ocupan parches pequeños. Por ejemplo los pícidos especialistas de hábitat, como el pico mediano Dendrocopos medius.

- Son vulnerables a la explotación humana por caza y la fragmentación del hábitat, que las hace más accesibles. Por ejemplo ciertos ungulados silvestres.

\section{CONCLUSIONES}

La importancia de los efectos de los procesos de fragmentación de hábitats sobre la vida silvestre les confiere un papel que no puede obviarse dentro de las políticas de conservación de la biodiversidad. Así, en el contexto internacional, existe una creciente toma de conciencia sobre la importancia de hacer frente a las graves consecuencias ecológicas derivadas de los procesos de fragmentación de hábitats, que ponen en cuestión la eficacia de las políticas clásicas de protección de la naturaleza. Se evidencia la necesaria integración en la toma de decisiones de incidencia territorial de criterios de prevención y corrección de aquellas transformaciones del paisaje que causan fragmentación de hábitats. Dicha integración debe realizarse tanto en las políticas horizontales territorial y ambiental, como en las políticas verticales sectoriales con incidencia territorial 
(urbanística, de transporte, agrícola, forestal, hidrológica, etc.). Todo ello hace que sea necesario el concurso de distintas disciplinas; biología, ecología, geografía, historia... y, a su vez, el trabajo de investigación, planificación y gestión de equipos multidisciplinares. Por tanto, se trata de un reto al que ha de enfrentarse la ordenación territorial en su conjunto.

\section{AGRADECIMIENTOS}

El presente artículo se enmarca dentro del proyecto, financiado por la Dirección de Biodiversidad y Participación Ambiental del Departamento de Medio Ambiente y Ordenación del Territorio del Gobierno Vasco, para el diseño de la red de corredores ecológicos de la Comunidad Autónoma del País Vasco, encargado a la empresa IKT, S.A. Agradecemos también la ayuda prestada por el Dr. Gabriel del Barrio, de la Estación Experimental de Zonas Áridas del CSIC (Consejo Superior de Investigaciones Científicas).

\section{BIBLIOGRAFÍA}

AlleE, W.C. ET AL. (1949): Principles of animal ecology. Saunders, Philadelphia.

ANDRÉN, H. (1995): «Effects of habitat fragmentation on birds and mammals in landscapes with differentes proportions of suitable habitat: a review». Oikos 71: 355366.

ANDRÉn, H. Y ANGELSTAM, P. (1988): «Elevated predation rates as an edge effect in habitat islands: experimental evidence». Ecology 69: 544-547.

ATAURI, J.A. Y DE LUCIO, J.V. (2001): «The role of landscape structure in species richness distribution of birds, amphibians, reptiles and lepidopterans in mediterranean landscapes». Landscape Ecology 16: 147-159.

BAKKER, J.D. Y WILSON, S.D. (2004): «Using ecological restoration to constrain biological invasion». Journal of Applied Ecology 41: 1058-1064.

BALDI, A. (1999): «Microclimate and vegetation edge effects in a reedbed in Hungary». Biodiversity and Conservation 8: 1697-1706.

BARRETT, G.W., FORD, H.A. Y RECHER, H.F. (1994): «Conservation of woodland birds in a fragmented rural landscape». Pacific Conservation Biology 1, 245-256.

BENDER, D.J., TISCHENDORF, L. Y FAHRIG, L. (2003): «Using patch isolation metrics to predict animal movement in binary landscapes». Landscape Ecology 18: 17-39.

BIERREGAARD, R.O. ET AL. (Eds.) (2001): Lessons from Amazonia: the ecology and conservation of a fragmented forest. Yale University Press.

BLONDEL, J. Y ARONSON, J. (1999): Biology and wildlife of the Mediterranean region. Oxford University Press, Oxford, UK.

BOWMAN, J., CAPPUCCINO, N. Y FAHRIG, L. (2002): «Patch size and population density: the effect of immigration behaviour». Conservation Ecology 6: 9.

BROOK, B.W. (2003): «Conservation biology: minimum sizes and habitat areas for viable wildlife populations». Center for Ecological Research, Kyoto University. CER News 79: 11-13. 
BROWN J.H. Y KoDRIC-BROWN, A. (1977): «Turnover rates in insular biogeography: effect of immigration on extinction». Ecology 58: 445-449.

BRUNA, E.M. Y OLI, M.K. (2005): «Demographic effects of habitat fragmentation on a tropical herb: life-table response experiments». Ecology 86: 1816-1824.

Burel, F. y BAUdry, J. (2002): Ecología del Paisaje. Conceptos, métodos y aplicaciones. Ediciones Mundi-Prensa, Madrid.

Chalfoun, A.D., Thompson, F.R. Y RATnASWAMY, M.F. (2002): «Nest predators and fragmentation: a review and meta-analysis». Conservation Biology 16: 306-318.

CONNOR, E. F., COURTNEY, A.C. Y YODER, J.M. (2000): «Individuals-area relationships: the relationship between animal population density and area». Ecology 81:734-748.

Crooks, K. Y SANJAYAN, M. (Eds.) (2006): Connectivity conservation. Cambridge University Press, Cambridge.

DEBINSKI, D.M. Y HoLT, R.D. (2000): «A survey and overview of habitat fragmentation experiments: a global survey and overview». Conservation Biology 14: 342-355.

DEL BARRIO, G. ET AL. (2000): «Aproximación para estimar la conectividad regional de las redes de conservación». En: V Congreso Nacional de Medio Ambente. Comunicaciones Técnicas. Colegio oficial de Físicos, Madrid: 1-17.

De Lucio, J.V., Atauri, J.A., SAstre, P. Y MArtíneZ, C. (2003): «Conectividad y redes de espacios naturales protegidos: del modelo teórico a la visión práctica de la gestión». En García Mora, M.R. (coord.) “Conectividad ambiental: las áreas protegidas en la cuenca mediterránea”. Junta de Andalucía: 29-54.

DeSRochers, A., HANSKI, I.K. Y SElOnEN, V. (2003): «Siberian flying squirrel responses to high- and low-contrast forest edges». Landscape Ecology 18: 543-552.

DIAMOND, J. (1975): «The island dilemma: lessons of modern biogeographic studies for the design of nature preserves». Biological Conservation 7: 129-146.

DÍAZ, J.A. ET AL. (2000): «Effects of forest fragmentation on the distribution of the lizard Psammodromus algirus». Animal Conservation 3: 235-240.

DÍAZ, J.A. ET AL. (2005): «Habitat fragmentation reduces reproductive investment and hatchling growth rate in Psammodromus algirus lizards». Conservation Biology 19: 1578-1585.

DÍAZ, M. ET AL. (1998): «Effects of pine plantations on bird communities of the Spanish plateaux: biogeography, landscape and vegetation effects». Journal of Applied Ecology 35: 562-574.

DÍAZ PINEDA, F. (2001): «Intensification, rural abandonment and nature conservation in Spain». En: BUNCE, R.G.H. ET AL. (eds.) Examples of European agri-environment schemes and livestock systems and their influence on Spanish cultural landscapes. Alterra, Wageningen: 23-38.

DIDHAM R.K. Y LAWTON, J.H. (1999): «Edge structure determines the magnitude of changes in microclimate and vegetation in tropical forest fragments». Biotropica 31: 17-30.

DONALD, P. F. (2004): «Biodiversity impacts of some agricultural commodity production systems». Conservation Biology 18: 17-37.

DOOLEY, L. Y BOWERS, M.A. (1998): «Demographic responses to habitat fragmentation: Experimental tests at the landscape and patch scale». Ecology 79: 969-980.

FAGAN W.F., CANTRELL, R.S. Y COSNER, C. (1999): «How habitat edges change species interactions». The American Naturalist 153: 165-182. 
FAHRIG, L. (2003): «Effects of habitat fragmentation on biodiversity». Annual Review of Ecology, Evolution and Systematics 34: 487-515.

FARINA, A. (1998): Principles and methods in landscape ecology. Chapman an Hall, London.

GASCON, C. ET AL. (1999): «Matrix habitat and especies richness in tropical forest remants». Biological Conservation 91: 223-229.

GILPIN, M.E. Y SOULÉ, M.E. (1986): «Minimum viable populations: processes of species extinction». En: Soulé, M.E. (ed.) Conservation biology, the science of scarcity and diversity. Sinauer Associates. Sunderland, Massachusetts: 19-34.

GONZÁlEZ BERNÁLDEZ F. (1981): Ecología y paisaje. Blume, Madrid.

HANSKI, I.A. Y GILPIN, M.E. (1997): Metapopulation biology: ecology, genetics, and evolution. Academic Press, San Diego.

HANSKI, I. (1999): Metapopulation ecology. Oxford University Press, Oxford, UK.

HARTLEY, M.J. Y HUNTER, M.J. (1998): «A meta-analysis of forest cover, edge effects and artificial nest predation». Conservation Biology 12: 465-469.

HENLE, K. ET AL. (2004): «Predictors of species sensitivity to fragmentation». Biodiversity and Conservation 13: 207-251.

HugGeTt, A.J. (2005): «The concept and utility of "ecological thresholds" in biodiversity conservation». Biological Conservation 124: 301-310.

JONGMAN, R.H.G. (2002): «Homogenisation and fragmentation of the European landscape: ecological consequences and solutions». Landscape and Urban Planning, 58, 211-221.

JULES, E.S. (1998): «Habitat fragmentation and demographic change for a common plant: Trillium in old-growth forest». Ecology 79:1645-1656.

LAURANCE, W.F. (1997): «Hyper-disturbed parks: edge effects and the ecology of iolated rainforest reserves in tropical Australia». En: Laurance, W.F. y Bierregaard, R.O. (eds.) Tropical forest remnants. Ecology, management, and conservation of fragmented communities. University of Chicago Press, Chicago: 71-83.

LAURANCE, W.F. (1990): «Comparative responses of five arboreal marsupials to tropical forest fragmentation». Journal of Mammalogy 71, 641-653.

LAURANCE, W.L. Y BIERREGAARD, R.O. (eds.). (1997): Tropical forest remnants. Ecology, management, and conservation of fragmented communities. University of Chicago Press, Chicago.

LAW, B.S. Y DICKMAN, C.R. (1998): «The use of habitat mosaics by terrestial vertebrate fauna: implications for conservation and management». Biodiversity and Conservation 7: 323-333.

LENS, L. ET AL. (1999): «Fluctuating asymmetry increases with habitat disturbance in seven bird species of a fragmented afrotropical forest». En Proceedings of the Royal Society of London, Biological Sciences 266: 1241-1246.

LEVINS, R. (1970): “Extinction”. En: Gerstenhaber, M. (ed.) Some mathematical problems in biology. American Mathematical Society, Providence: 75-107.

LINDENMAYER, D.B. Y FRANKLIN, J.F. (2002): Conserving forest biodiversity: a comprehensive multiscaled approach. Island Press, Washington.

LOMOLINO, M.V. (2000): «Ecology's most general, yet protean pattern: the species-area relationship». Journal of Biogeography 27: 17-26. 
LOMOLINO, M.V. Y WEISER, M.D. (2001): «Towards a more general species-area relationship: diversity on all islands, great and small». Journal of Biogeography 28: 431-445.

LOVEJOY T.E. ET AL. (1986): «Edge and other effects of isolation on Amazon forest fragments». En: SoulÉ, M.E. (ed.), Conservation biology: the science of scarcity and diversity. Sinauer Associates, Mass: 257-325.

LUCK, G.W. (2005): «An introduction to ecological thresholds». Biological Conservation 124: 299-300.

MACARTHUR, R.H. Y WILSON, E.O. (1967): The theory of island biogeography. Princeton University Press, Princeton, New-Yersey.

MANDUJANO, A. Y ESTRADA, A. (2005): «Umbrales de área y distancia de aislamiento y presencia de monos aulladores, Alouatta palliata, en fragmentos de selva en Los Tuxtlas, México». Universidad y Ciencia $\mathrm{N}^{\circ}$ Especial II: 11-21.

MAtlack, G. Y LiTVAitis, J. (1999): «Forest edges». En Hunter, M.L. (ed.) Maintaining biodiversity in forest ecosystems. Cambridge University Press. Cambridge: 210233.

MERRIAM, G. (1984): «Connectivity: a fundamental ecological characteristic of landscape pattern». En: BRANDT, J. Y AGGER, P. Proceedings of the First International Seminar on Methodology in Landscape Ecological Research and Planning. Roskilde Universitetsforlag GeoRuc, Roskilde, Dinamarca: 5-15.

MÚGICA, M., ET AL. (2002): Integración territorial de espacios naturales protegidos y conectividad ecológica en paisajes mediterráneos. Consejería de Medio Ambiente, Junta de Andalucía, Sevilla.

MurCiA, C. (1995): «Edge effects in fragmented forests: implications for conservation». Trends in Ecology and Evolution 10: 58-62.

NAVEH, Z. (1994): «From biodiversity to ecodiversity: landscape ecology approach to conservation and restoration». Restoration Ecology 2:180-189.

Noss, R.F. Y CsUTI, B. (1994): «Habitat fragmentation». En: MEFFE, G.K. Y CARROLL, C.R. (Eds.) Principles of Conservation Biology. Sinaur Associates Inc., Sunderland, Massachusetts: 237-264.

ODUM, E.P. (1958): Fundamentals of ecology. Saunders, Philadelphia.

OPDAM, P. (1991): «Metapopulation theory and habitat fragmentation: a review of holarctic breeding bird studies». Landscape Ecology 5: 93-106.

PALIK, B.J. Y MURPHY, P.G. (1990): «Disturbance versus edge effects in sugarmaple/beech forest fragments». Forest Ecology and Management 32: 187-202.

PINO, J. ET AL. (2000): «Landscape structure and bird species richness: implications for conservation in rural areas between natural parks»». Landscape and Urban Planning 9: $5-48$.

PRESTON, F.W. (1962): «The canonigal distribution of commonness and rarity: Part I». Ecology 43: 185-215.

PRIMACK, R. (2002): Essentials of Conservation Biology. Sinauer Associates, Sunderland.

Pulliam, H.R. (1988): «Sources, sinks and population regulation». The American Naturalist 132: 652-661. 
RADFORD, J.Q., BENNETT, A.F. Y CHEERS, G.J. (2005): «Landscape level thresholds of habitat cover for woodland dependent birds». Biological Conservation 124: 317 337.

RIVARD, D.H. ET AL. (2000): «Species richness and changes in species composition in Canada's national parks: With-in park habitat and the regional context». Conservation Biology 14: 1099-1110.

RosenzWEIG, M.L. (1995). Species diversity in space and time. Cambridge University Press, Cambridge.

SANTOS, T. Y TELLERÍA, J.L. (1998): «Efectos de la fragmentación de los bosques sobre los vertebrados en las mesetas ibéricas». Organismo Autónomo Parques Nacionales, Ministerio de Medio Ambiente, Madrid.

SANTOS, T. Y TELLERÍA, J.L. (2006): «Pérdida y fragmentación del hábitat: efecto sobre la conservación de las especies». Ecosistemas 2: 3-12.

SCHIEGG, K. (2000): «Effects of dead wood volume and connectivity on saproxylic insect species diversity». Ecoscience 7: 290-298.

SCHTICKZELLE, N. Y BAGUETTE, M. (2003): «Behavioural responses to habitat patch boundaries restrict dispersal and generate emigration-patch area relationships in fragmented landscapes». Journal of Animal Ecology 72: 533-545.

SCHUlTZ, C.B. Y CRONE, E.E. (2005): «Patch size and connectivity thresholds for butterfly habitat restoration». Conservation Biology 19: 887-896.

SHAFFER, M.L. (1981): «Minimum population sizes for species conservation». BioScience 31: 131-134.

SHAFFER, M.L. (1987): «Minimum viable population: coping with uncertainty». En: Soulé, M. (Ed.) Viable Population for Conservation. Cambridge University Press, Cambridge.

SimBERLOFF, D. (1982): «Big advantages of small refuges». Natural History 91: 6-14.

SIMBERLOFF, D.S. Y L.G. ABELE. (1976): «Island biogeography theory and conservation practice». Science 191: 285-286.

STEPHENS, S.E. ET AL. (2003): «Effects of habitat fragmentation on avian nesting success: a review of the evidence at multiple spatial scales». Biological Conservation 115: 101-110.

SUAREZ-SEOANE S., OSBORNE P.E Y BAUDRY J. (2002): «Responses of birds of different biogeographic origins and habitats to agricultural land abandonment in northern Spain». Biological Conservation 105: 333-344.

TAYLOR P.D. ET AL. 1993. "Connectivity is a vital element of landscape structure». Oikos 68: 571- 573.

TELLERÍA, J.L. Y SANTOS, T. (1999): «Distribution of birds in fragments of Mediterranean forest: the role of ecological densities». Ecography 22. 13-19.

TELLERÍA, J.L. (2001): «Gestión forestal y conservación de la fauna de vertebrados». En: CAMProdon, J. y PlANA, E. (Eds.) Conservación de la biodiversidad y gestión forestal. Su aplicación en la fauna vertebrada. Edicions Universitat de Barcelona, Barcelona: 119-133.

TISCHENDORF, L. ET AL. (2005): «Mechanisms affecting population density in fragmented habitat». Ecology and Society 10: 7.

TOMS, J.D. Y LESPERANCE, M.L. (2003): «Piecewise regression: a tool for identifying ecological thresholds». Ecology 84: 2034-2041. 
TUCKER, G.M. (1997): «Priorities for bird conservation in Europe: the importance of the farmed landscape». In: Pain, D.J. y Pienkowski, M.W. (Eds.) Farming and Birds in Europe: The Common Agricultural Policy and its Implications for Bird Conservation. Academic Press, London: 79-116.

TURNER, M.G. Y GARDNER, R.H. (Eds.). (1991): Quantitative Methods in Landscape Ecology. Springer-Verlag, New York, USA.

WIENS, J.A., CRAWFORD, C.S. Y GOSZ, J.R. (1985): «Boundaruy dynamics: a conceptual framework for studying lanscape ecosystems». Oikos 45: 421-427.

WiLCOVE, D.S. (1985): «Nest predation in forest tracts and the decline of migratory songbirds». Ecology 66: 1211-1214.

Williams, C.B. (1964): Patterns in the balance of nature and related problems in quantitative biology. New York Academy Press, New York.

WILLSON, M.F. ET AL. (2001): «Patterns of predation risk and survival of bird nests in a chilean agricultural landscape». Conservation Biology 15, 447-456.

WHITTAKER, R. (1998): Island biogeography. Ecology, evolution and conservation. Oxford University Press.

WITH, K.A. ET AL. (2002): «Threshold effects of landscape structure on biological control in agroecosystems». Ecological Applications 12: 52-65.

WITH, K.A. Y CRIST, T.O. (1995): «Critical thresholds in species' responses to landscape structure». Ecology 76: 2446-2459.

WITH, K.A. y KING, A. W. (1999): «Dispersal success on fractal landscapes: a consequence of lacunarity thresholds». Landscape Ecology 14: 73-82.

WOLF, M. Y BATZLI, G. O. (2002): «Effects of forest edge on populations of whitefooted mice Peromyscus leucopu». Ecography 25: 193-199. 